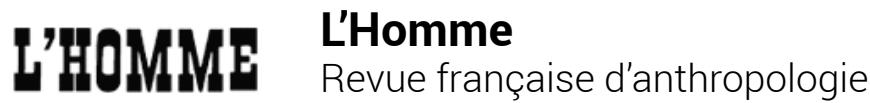

187-188 | 2008

Miroirs transatlantiques

\section{Michel Wieviorka, ed., Les Sciences sociales en mutation}

\section{Corinne Delmas}

\section{(2) OpenEdition \\ 1 Journals}

Édition électronique

URL : https://journals.openedition.org/lhomme/20822

DOI : 10.4000/lhomme.20822

ISSN : 1953-8103

Éditeur

Éditions de l'EHESS

\section{Édition imprimée}

Date de publication : 3 octobre 2008

Pagination : 507-509

ISBN : 978-2-7132-2186-6

ISSN : 0439-4216

\section{Référence électronique}

Corinne Delmas, « Michel Wieviorka, ed., Les Sciences sociales en mutation », L'Homme [En ligne], 187-188 | 2008, mis en ligne le 16 décembre 2008, consulté le 24 avril 2022. URL : http:// journals.openedition.org//homme/20822 ; DOI : https://doi.org/10.4000//homme.20822

Ce document a été généré automatiquement le 24 avril 2022.

(c) École des hautes études en sciences sociales 


\title{
Michel Wieviorka, ed., Les Sciences sociales en mutation
}

\author{
Corinne Delmas
}

\section{RÉFÉRENCE}

Michel WIEVIORKA, ed., Les Sciences sociales en mutation. Avec la collab. d'Aude Debarle \& Jocelyne Ohana. Auxerre, Sciences Humaines, 2007, 624 p.

1 CET OUVRAGE est issu d'un colloque qui s'est tenu à Paris en mai 2006, à l'occasion des vingt-cinq ans du Centre d'analyse et d'intervention sociologique (CADIS) fondé par Alain Touraine et dirigé par Michel Wieviorka. Il fédère cinquante auteurs, pour la plupart membres du CADIS, et rassemble quarante-huit contributions autour de la problématique du renouvellement des sciences sociales à l'orée du xxi siècle, traitant aussi bien de leurs objets et paradigmes que de leurs méthodes et outils d'analyse. Huit grands thèmes regroupent ces textes : la place du sujet; la mondialisation, le temps et l'espace ; les concepts, disciplines et carrefours ; les changements dans les paradigmes, continuités et retours; la religion; le travail, l'emploi et l'entreprise; l'histoire; les sentiments d'injustice, la démocratie et l'imaginaire social.

2 Si les auteurs sont majoritairement des sociologues, les autres sciences sociales ne sont pas pour autant ignorées, l'ouvrage comprenant aussi des textes de démographes, d'historiens, d'économistes, de géographes, d'anthropologues, de juristes, de politistes et de philosophes... Il s'agit également pour les sciences sociales de dialoguer avec les sciences cognitives et les sciences dites " exactes ", d'où la participation d'un médecin biologiste spécialiste d'immunologie et d'un philosophe des sciences cognitivistes.

3 Selon Michel Wieviorka, tout aurait changé : les méthodes, théories et paradigmes pivots des sciences humaines et sociales seraient, aujourd'hui, déstructurés, avec la fin du marxisme, les remises en cause du structuralisme, l'éloignement des prétentions à proposer des théories générales et de l'ambition de totalité, question particulièrement abordée par Alain Blum qui en analyse les conséquences méthodologiques à partir de 
ses recherches sur l'histoire russe et soviétique. Ainsi, le cadre des sciences sociales ne serait plus national mais mondial, d'où la nécessité de saisir l'enjeu de la globalisation sur plusieurs fronts (flux migratoires, culture, droit)... Ces évolutions participent au renouvellement des méthodes et des objets, suscitant, par exemple en histoire, comme le souligne Olivier Pétré-Grenouilleau, un intérêt pour une «histoire globale comme entreprise destinée à essayer d'éclairer, sinon la totalité, du moins le plus grand nombre de facettes d'un même objet» (p.530), générant également "de nouvelles opportunités de lecture et d'analyse " et la prise en compte de thèmes longtemps délaissés en France comme la violence de la guerre, abordée par Stéphane AudoinRouzeau. De même, la montée du sujet contribue à ouvrir de nouveaux champs de recherche dont celui du corps, étudié par Georges Vigarello, ou du mourant et de son «impossible statut», traités par Philippe Bataille. Elle suscite surtout de nombreux défis, dont une nécessaire remise en cause d'opérations de catégorisation procédant "très souvent d'un acte de violence », selon François Laplantine (p. 47), la prise en compte de "la combinaison des contraires chez l'individu et dans l'interaction », du " rôle paradoxal et du rôle ambivalent » (p.370), en particulier dans le cas du genre étudié par Christine Castelain Meunier, l'intérêt pour l'expérience individuelle et les « distorsions » entre celle-ci et « la représentation d'une vie sociale organisée » (p. 566) analysées par François Dubet. Un renouvellement des méthodes s'impose et François de Singly propose de réhabiliter l'individu concret, de privilégier les portraits mais aussi d'élargir la vision objective des individus en prenant en compte l'autrui significatif, l'individu pouvant être pensé aujourd'hui comme un «individu réseau " (p.69). Le point de vue de la sociologie clinique est apporté par Vincent de Gaulejac pour questionner la notion même de sujet dont «la sollicitation permanente [...] fait problème dans la mesure où elle participe de l'exigence de performance qui caractérise notre société » (p.80). Selon Olivier Cousin, "l'exaltation de la subjectivité, de la réussite, de la performance et de la prise de risque revient à une forme d'autoaliénation » dans une société et un monde du travail qui, ayant érigé en "principe la souplesse et la flexibilité » (p.482), sont générateurs d'angoisse et ne laissent guère d'espace à l'acteur. Richard Sennett souhaite pour sa part que « la sociologie s'intéresse de plus près au récit comme un des nombreux outils dont dispose l'individu pour se comprendre » (p. 446), comme en attestent les «stratégies narratives» des salariés en situation de précarité analysées ici. L'expérience vécue fait donc l'objet d'un intérêt croissant de la part des chercheurs. À propos du racisme, Alexandra Poli montre combien cette perspective remet en question la passivité habituellement attribuée aux victimes et pose la question de la compréhension et de la place de leur subjectivité. Et un tel changement de perspective "représente un enjeu de taille pour la culture politique républicaine de la France » (p. 107). Elle n'est pas sans conséquence pratique, en particulier en matière de lutte contre les discriminations. La question sous-jacente de l'effectivité des sciences sociales, des liens entre science et politique, théorie et pratique, voire de l'engagement du chercheur est récurrente. Jean-Pierre Dozon aborde ainsi la question de la réflexivité et interroge le «malaise» de sciences sociales s'accommodant difficilement "des retours ou des manifestations intempestives de mouvements religieux et d'expériences du passé dont elles pensaient avoir peu ou prou la maîtrise " (p. 377) dans un monde sécularisé dont les contributions de Laurence Moore, de Hans Joas et de Clifford Geertz soulignent la relativité et l'ambiguïté. Alain Blum interpelle le lecteur sur la «déconnexion, non reconnue, entre les outils du politique et ceux des sciences sociales » et le décalage qui augmenterait «entre deux 
mondes [...] le politique et les sciences sociales » (p. 364). Wiktor Stoczkowski montre, à partir de sa propre expérience, combien «la connaissance anthropologique pouvait être, parfois du moins, contraire aux impératifs de l'action politique et de la conviction morale » (p. 296). Il choisit ainsi de ne pas publier les résultats d'une enquête qu'il avait faite sur les partisans convaincus et la police politique d'un régime totalitaire de l'Est dans la mesure où ils étaient jugés inacceptables par les acteurs et peu aptes «à resserrer nos rangs et à mobiliser nos énergies autour d'une vision clairement négative de nos ennemis" (ibid.). Ce sont ainsi les questions de la conciliation entre la connaissance et l'éthique, la science et l'engagement, et de l'éventuel renoncement du scientifique qui se posent pour des sciences sociales devant affronter une difficulté axiologique: "nous n'avons pas été suffisamment attentifs au fait que les valeurs morales qui orientent la recherche sont de nature à imposer des limites considérables au savoir dont les chercheurs espèrent obtenir des répercussions éthiques et politiques » souligne Wiktor Stoczkowski (p. 297). La pression extérieure sur le travail de l'historien est également abordée par Annette Wieviorka, à propos de « la tyrannie de la mémoire " à laquelle ont été confrontés les historiens de la Seconde Guerre mondiale " écrivant une histoire sous la surveillance parfois peu amène des acteurs " (p. 515). Craig Calhoun estime qu'« il existe dans le monde d'aujourd'hui un besoin impérieux de ce que nous pourrions appeler une "science sociale du réel" » et qu'« il est vital de produire des connaissances qui répondent aux problèmes publics cruciaux et de les proposer assez vite pour éclairer les débats ». Mais, selon cet auteur, «nous ne sommes pas bons à cet exercice " (p. 155). "Viser un engagement public plus efficace des sciences sociales exige, en bref, leur transformation»(p.156), en particulier promouvoir une perspective historique afin « de saisir la nature et la signification de ce qui se passe" (p.157) et être attentif aux "modifications dans la manière dont la réalité est structurée par les catégories de connaissances qui sont aussi des catégories d'action pratique» (p.159) comme par exemple l'imaginaire de l'urgence étudié ici. Charles Taylor montre l'utilité d'une telle discussion en termes de répertoires et d'imaginaires sociaux pour la compréhension de l'avènement de la démocratie et de la souveraineté des peuples. Selon Danièle Hervieu-Léger, ces multiples défis impliquent le dialogue des sciences sociales avec les autres sciences. Elle recense cependant les difficultés d'un tel dialogue, l'appel à l'interdisciplinarité fonctionnant "comme une sorte d'utopie de l'unité retrouvée de tous les savoirs, perpétuellement rêvée et jamais pratiquée, ce qui est le lot ordinaire de l'utopie» (p.240). Plusieurs phénomènes hypothéqueraient une telle interdisciplinarité: «reconduction massive d'une hiérarchie des dignités scientifiques subordonnant [...] les sciences humaines et sociales aux sciences de la nature ", intériorisation par les chercheurs en sciences sociales « de l'évidence selon laquelle leurs disciplines souffrent d'un déficit quasi-ontologique d'épistémologie » (p. 241), incertitude identitaire qui en résulte... Toutefois, les sciences humaines et sociales pourraient bénéficier aujourd'hui de deux grandes tendances: d'une part, la montée de la thématique de la complexité « qui favorise la convergence et l'échange entre des entreprises intellectuelles très variées» et, d'autre part, l'émergence d'une «nouvelle pensée de l'individu » mettant en question les séparations longtemps établies entre les processus physiologiques, mentaux et sociaux (pp. 243-244). C'est dire combien les perspectives et les champs de recherches à venir s'avèrent nombreux et fructueux pour ces sciences, au centre des débats et ancrés dans l'actualité... 